\title{
Novel synthesis techniques for preparation of ultrahigh-crystalline vanadyl pyrophosphate as a highly selective catalyst for n-Butane oxidation.
}

\begin{abstract}
The vanadyl hydrogen phosphate hemihydrate (VOHPO40.5H2O), with well-defined crystal size, has been successfully synthesized for the first time, using a simple one-step solvothermal process that was free of surfactants and water and had a short reaction time and low temperature. The synthesis was performed via the reaction of V2O5 and H3PO4 with an aliphatic alcohol (1-propanol or 1-butanol) at high temperatures $(373,393$, and $423 \mathrm{~K}$ ) in a high-pressure autoclave. The mixture of reactions directly gave the VOHPO40.5H2O, which is a valuable commercial catalyst precursor for the selective oxidation of n-butane to maleic anhydride. The catalyst precursors were dried by microwave irradiation. The reaction conditions (by varying the reducing agent and reaction temperature) were used further for optimization of the crystallite size, surface area, morphology, and activity of the nanostructure of vanadium phosphate oxide [(VO)2P2O7] catalyst. This new method significantly reduced the preparation time and lowered the production temperature $(50 \%)$ of catalyst precursor (VOHPO40.5H2O), when compared to conventional hydrothermal synthesis methods. The as-prepared (VO)2P2O7 catalyst under various conditions exhibited remarkably different physical and chemical properties, indicating the potential of the suggested method in tuning the crystalline structure and surface area of (VO)2P2O7 to improve its catalytic performance. It was found that the length of the carbon chain in an alcohol and reaction temperature in the solvothermal condition had a great impact on the chemical and physical properties of resulting catalysts. Interestingly, there was no trace of $\mathrm{VO}(\mathrm{H} 2 \mathrm{PO} 4) 2$, which is an impurity noted to be readily formed under solvothermal preparation conditions. The precursors and catalysts were characterized using a combination of powder X-ray diffraction (XRD), Brunauer-Emmett-Teller (BET) surface area measurement, scanning electron microscopy (SEM), and temperature-programmed reduction in hydrogen (H2-TPR). A correlation between the surface area of the catalyst and the activity was observed. Finally, the yield of maleic anhydride was significantly increased from $21 \%$ for conventional catalyst to $38 \%$ for the new solvothermal catalyst.
\end{abstract}

Keyword: solvothermal; Butane oxidation; Maleic anhydride. 